\title{
Salutogenesis and the Mental Health of First Responders
}

\author{
Avishai Antonovsky
}

\section{Introduction}

Pictures of beautiful sunsets, crystal-clear lakes surrounded by lovely forests, snowy mountains, rocky canyons, pastoral meadows, and exotic beaches often create an emotional, sometimes even sensory, experience of tranquility and peace of mind. Likewise, a quiet evening spent in one's living room with a few good friends, a glass of wine, and a burning log in the fireplace is a perfect recipe for what would be termed "hygge" in Danish - the feeling of coziness, togetherness, comfort, and contentment.

However, while at times, nature can be a source of relaxation, health, and well-being (Bodman, 2017), and being in a group of people whom you know and value can produce feelings of joy and security, at other times the opposite may occur. Natural and human surroundings can be the places where disastrous and fatal events occur. During the decade between 2008 and 2018, an annual average of 1.35 million people around the globe died in automobile accidents (World Health Organization, 2018); more than 20,000 were killed in terrorist attacks (Ritchie et al., 2019); about 140,000 lost their lives in fires (Ritchie \& Roser, 2019a); and 60,000 fatalities were caused by natural disasters each year (Ritchie \& Roser, 2019b). As of September 2021, the death toll of the coronavirus (COVID-19) pandemic has crossed 4,500,000 victims worldwide (www.worldometers.info).

These unfortunate circumstances will be the context of the discussion in this chapter. However, the focus will not be on those who died, but rather on those who were there to help and save lives: the first responders. First responders (FRs) are people who are first to arrive and provide care at emergency sites like fires, terrorist attacks, or natural disasters. FRs may be professionals such as firefighters, paramedics, body handlers, search and rescue teams, mental health professionals, forensic examiners, or police officers. Still, they

A. Antonovsky $(\varangle)$

Department of Health and Well-being, Medical Corps,

Israel Defense Forces, Ramat-Gan, Israel

e-mail: Antonovsky.soc@gmail.com may also be nonprofessional volunteers in their communities. Or, in the words of Allison McCullough from the US First Responders Association, "A first responder is any individual who runs toward an event rather than away" (USFRA, 2019).

There is a large body of literature on psychopathological consequences among FRs and the treatment of them following disastrous or potentially traumatic events. Despite this, structured programs for mental preparation of FRs before these events are relatively scarce, and most of them are pathogenically oriented (i.e., aimed at preventing psychopathology). In this chapter, I will first review the mental effects that repeated exposure to emotionally challenging scenes may have on FRs, and some protocols used following disasters for what has been generally termed "helping the helpers." Then, I will present what I have found about programs designed to enhance FR's mental fitness. Finally, I will suggest an outline for a salutogenic model of mental preparation for FRs, based on what has been done in the Israel Defense Forces (IDF) in the last few years.

\section{Mental Hazards in the Work of First Responders}

As part of my job as civilian researcher in the IDF's Department of Health and Well-being, I worked with a military unit of body handlers that is in charge of taking care of bodies and bodily remains of people who were killed in unnatural circumstances (automobile accidents, terrorist attacks, fires, etc.). One of these FRs told me about his 2-year-old daughter and how he sings to her every evening before she goes to sleep. "But," he said, "I cannot bring myself to cover her with her blanket and tuck her in." Covering someone with a blanket is part of what he does at work, and this physical act is something that he cannot manage, emotionally, to transfer from one context to another.

Most of the literature about crisis intervention, which is where FRs are usually needed, focuses on the needy - those 
who get hurt physically or mentally. Many intervention models for these circumstances have been developed all over the world. This is not the main topic of this chapter, but I will mention just two, to set the context for the reader.

Over the past 30 years, Israel has experienced several bomb and missile attacks on its northern and southern borders. The work of mental health professionals during these events - usually social workers - has been important in the treatment of mentally injured people, usually suffering from anxiety. Two well-known models for crisis intervention by FRs that were developed in Israel are BASIC-Ph (Lahad, 2017), which is intended for professional FRs, and the Six-C model (Farchi et al., 2018; Hantman \& Farchi, 2015), which is intended for nonprofessionals who find themselves with victims of a disaster or catastrophic event. Farchi's Six-C model addresses the need to provide immediate standardized mental first aid to a person who is exhibiting an acute stress reaction (ASR) and to shift the person from being a helpless victim of functional breakdown to becoming a coping survivor. This can be in the face of a terrorist attack, or a flood, or a fire, or any other unexpected human or natural disaster. Theoretically, the protocol is based on the concepts of sense of coherence (SOC) (Antonovsky, 1979, 1987), self-efficacy (Bandura, 1997), and hardiness (Kobasa, 1979), as well as on neuropsychological processes involving the relation between the limbic system and the prefrontal cortex during stressful events (Farchi et al., 2018). In a few meetings I had with Farchi over the years, he would always mention the salutogenic model and how important it is for health promotion. Still, his Six-C model is focused on emergency treatment of ASR to prevent the reaction from becoming a disorder. In other words, the Six-C model, while being quite helpful (Farchi et al., 2018; Svetlitzky et al., 2019), is about diagnosis and treatment, not about salutogenic health promotion. People with ASR are those who experience functional breakdown during an emergency - characterized by detachment, shaking, loneliness, confusion, emotional shock, and helplessness. This is a normal reaction to an abnormal situation and can occur to anyone. If 48-72 hours pass without treatment, people who have exhibited an ASR may develop an acute stress disorder (ASD). ASD is diagnosed by the existence of a variety of posttraumatic stress disorder (PTSD) symptoms (intrusion, avoidance, and alterations in cognition, mood, and arousal; see American Psychiatric Association, 2013). For some people, spontaneous recovery may occur. For others, ASD may evolve over a few months, stabilize, and crystalize into chronic PTSD.

For FRs to witness or treat people with an ASR, or to see death and serious bodily injury repeatedly, is emotionally draining. Thus, FRs are prone to mental difficulties in two ways. One is through experiencing direct trauma because of being exposed to a life-threatening situation of self or others, and the other is by the recurring witnessing of death and destruction. Either way, psychological distress and PTSD can develop within emergency FRs (e.g., paramedics, body handlers, police investigators, and firefighters; see Donnelly, 2012; Ramey et al., 2016; Smith et al., 2018; Zerach \& Levin, 2015, to mention just a few). The occurrence of these outcomes is usually related to the frequency of exposure and severity of exposure to critical incidents (Weiss et al., 2010). The prevalence of PTSD among FRs can reach $10 \%$ and is relatively high in developing countries and among ambulance personnel. This is very high (up to 20 times as much) compared to the prevalence in the general adult population (American Psychiatric Association, 2013; Berger et al., 2012; Bezabh et al., 2017). For example, Bezabh et al. reported that $19.9 \%$ of FRs working in the Fire and Emergency Control and Prevention Authority in Addis Ababa, Ethiopia, were diagnosed with PTSD while still on their job. In a recent paper on the prevalence of PTSD among Canadian FRs, the numbers are even higher (Szeto et al., 2019). Considering these data, it is worth supplying the readers with the definition of trauma, which is the first and necessary criterion for the diagnosis of PTSD and is relevant to FRs (see criterion A4) (American Psychiatric Association, 2013, p. 271):

A. Exposure to actual or threatened death, serious injury, or sexual violence in one (or more) of the following ways:

1. Directly experiencing the traumatic event(s).

2. Witnessing, in person, the event(s) as it occurred to others.

3. Learning that the traumatic event(s) occurred to a close family member or close friend. In cases of actual or threatened death of a family member or friend, the event(s) must have been violent or accidental.

4. Experiencing repeated or extreme exposure to aversive details of the traumatic event(s) (e.g., first responders collecting human remains; police officers repeatedly exposed to details of child abuse).

Note: Criterion A4 does not apply to exposure through electronic media, television, movies, or pictures unless this exposure is work related.

In addition to PTSD, FRs are at higher risk than the general population for anxiety disorders, depression, alcohol abuse, early retirement, divorce, and suicide (Brondolo et al., 2012; Haugen et al., 2017). The reported rates of these cases are probably lower than the actual numbers because shame, stigma, and unawareness prevent many FRs from accessing mental health services (Haugen et al., 2017; Heyman et al., 2018).

The COVID-19 pandemic provided a unique and unusual example of FRs' exposure to threat. Several doctors and 
nurses were assigned to take care of coronavirus patients under circumstances that are very different from what they are accustomed to in their routine work. A considerable number of studies have already found that these healthcare professionals experience high levels of anxiety, depression, burnout, and sense of threat, related to their possible exposure to the virus (e.g., Adams \& Walls, 2020; Chen et al., 2020; Lai et al., 2020; Tan et al., 2020). Similar findings were reported in an Israeli study among military reserve rescue teams of the Home Front Command. They were recruited and assigned to reinforce Israel's national emergency medical service at its operational call center (Avishai Antonovsky et al., 2021). These are examples of a unique situation in which healthcare professionals were acting as FRs on assignments which they had never been trained for, accompanied by a great deal of uncertainty regarding the proper treatment of patients and the personal security measures that need to be taken.

Like FRs on the site of an emergency, mental health workers in a clinic who treat individuals who have suffered trauma are at risk themselves for compassion fatigue. Compassion fatigue (or the earlier term used, secondary traumatic stress disorder) refers to the influence on mental health professionals of the therapeutic encounter or intervention with victims of disaster suffering PTSD (Lahad, 2000). Here, too, the literature concentrates on interventions for helping the helpers in terms of treatment targeted to alleviate the effects of such mental difficulties that mental health workers go through (Bercier, 2013). Likewise, Brondolo and her colleagues offered a model used to generate worksite intervention strategies to reduce the risk for PTSD among FRs (Brondolo et al., 2008).

In the context of mental preparation and intervention programs, there is a common use of the term "resilience." For reasons I will shortly bring forward, in the current context, I prefer the term "mental fitness" over "resilience." Conceptually, the construct of mental fitness is like that of physical fitness: it is something that can be learned, trained for, and preserved by exercise (see Bolier et al., 2013, and Robinson, 2014, for similar conceptualizations). It is multifaceted in the sense of having a reservoir of various resistance resources and can be measured at a personal as well as a group level. Mental fitness is defined as follows:

a learned and conservable competency which is a product of the social, emotional, cognitive, and physiological capacities of a person or a group to cope successfully with mental challenges (Avishai Antonovsky et al., 2017).

Enhancing mental fitness is therefore the process of building mental strength to withstand and cope well with mental challenges. This is different from resilience. Resilience, although intuitively understood, seems to be an elusive concept. As Windle (2011, p. 152) argued, on a conceptual level, "the complexities of defining what appears to be the relatively simple concept of resilience are widely recognized, especially within the behavioural sciences." On an empirical level, she added, "This creates considerable challenges when developing an operational definition of resilience; definitional variation leads to inconsistencies relating to the nature of potential risk and protective processes, and in the estimates of prevalence." According to Windle, resilience is considered a psychological trait, and it seems that the one characteristic of resilience which is agreed upon is its meaning of being able to "bounce back" in the face of adversity. As Zamorski (2008, p. 7) stated, "Psychological resilience $\ldots$ is defined as the "sum total of psychological processes that permit individuals to maintain or return to previous levels of well-being and functioning in response to adversity'." Eshel et al. (2017), who studied individual attributes as predictors of resilience, opened their article with three different definitions of resilience, after which they offer their own. All four definitions have in common the idea of functioning (or bouncing back) following adversity or a traumatic event. Hence, an operational definition of resilience must include the existence of adversity from which bouncing back can be measured, just as PTSD can be diagnosed only if a traumatic event has occurred. Mental fitness, on the other hand, is not considered a trait, and it can be trained for. It is not narrowed to a unidimensional psychological construct and can be operationalized without the need for adversity to occur. While resilience is important to have once a person has experienced a (potentially) traumatic event, mental fitness is important to have before such an event occurs. Taken to the hypothetical extreme, it can be argued that a person with a very high degree of mental fitness does not have to worry about being resilient.

The concept of mental fitness shares two important characteristics with SOC: one is having cognitive, behavioral, and emotional aspects (which parallel SOC's components of comprehensibility, manageability, and meaningfulness). The other is that both are conceptualized and can be operationalized at both the individual and the group levels. Mental fitness is more specific and task oriented than SOC, but the stronger the people's SOC, the easier it will be for them to develop and maintain a high level of mental fitness. For example, in the study described earlier of military rescue soldiers in Israel's national emergency medical service during the COVID-19 pandemic, SOC was the best predictor of (and inversely related to) sense of threat, state anxiety, and burnout (Avishai Antonovsky et al., 2021). In that study, it was also found that a subgroup of soldiers who had been given structured mental preparation based on salutogenic principles of providing information, strengthening resistance resources, and emphasizing meaningfulness exhibited less burnout and a higher degree of subjective well-being than those who had not received such preparation (Danon et al., 2020). Likewise, in a study among volunteer body handlers 
who belonged to the Israeli organization ZAKA (Hebrew acronym for "identification of disaster victims"), SOC was found to have a buffering effect on posttraumatic stress symptoms and burnout (Zerach \& Levin, 2015). Zerach and Levin recommended that SOC "should be taken into account in the process of recruitment and training of body handlers" (p. 2).

As mentioned earlier, mental preparation for FRs is not common. The literature on "helping the helpers" is mostly devoted to postevent treatment. Large-scale, theoretically driven, and evidence-based programs intended to proactively prepare FRs for coping with mentally challenging and emotionally draining situations are hard to find.

Searching the literature, I found a few papers that recommended "pre-incident training" as a prevention technique for traumatic stress among FRs (e.g., Cochran \& Bardi, 2010). Still, this kind of training is usually limited in its scope and centers on generic forms of psychoeducation. One example is the seemingly promising "Road to Mental Readiness" (R2MR) program, developed by Canada's Department of National Defence (Szeto et al., 2019). The R2MR program is intended to reduce stigma and negative attitudes toward those struggling with poor mental health (thus removing barriers from seeking care), to provide FRs with a broad understanding of mental health and wellness, and to use a mental health continuum scale as a self-assessment tool for defining mental health in themselves and others. The R2MR program is usually carried out in one 4-hour psychoeducational session. Szeto and his colleagues reported that the R2MR intervention has increased self-reported resiliency and decreased stigmatizing attitudes at a 3-month follow-up. However, Carleton et al. (2018) studied long-term effects of the one-session $\mathrm{R} 2 \mathrm{MR}$ intervention on a series of outcome measures and reported that besides a small short-term decrease in stigmatic attitudes toward mental illness, there were "no statistically significant changes in symptoms of depression, anxiety, stress, posttraumatic stress, and alcohol use, at any follow-up time point, following the training intervention" (p. 521). Although I read a few sources besides the ones cited here, I could not uncover any sound theoretical foundation for the development of the R2MR program, besides the logical assumption that psychoeducation on mental health and stigma has the potential of increasing help-seeking behavior.

I agree with Szeto et al. (2019) who wrote "it seems evident that implementation of a program that addresses resilience and stigma reduction in first responders is warranted and may offer positive impact (e.g., improved mental health)" (p. 19S). However, what they offer in the R2MR program is mainly treatment oriented and may not consider all aspects of what is needed to increase mental fitness, as part of a general health promotion approach toward helping the helpers.

Another example of what at first seemed like a salutogenic approach to enhancing FRs' mental fitness is a web- based training program for medical examiners, which consists of self-paced instruction modules (Brondolo et al., 2017). The training is aimed at developing emotion regulation skills, as a means of reducing the risk of mental problems such as depression and PTSD. Brondolo and her colleagues appeared to be adopting a positive psychology approach when in the last paragraph of their paper they wrote: "a combined program of research and intervention can provide insight into strategies for strengthening psychological capital among the first responder community" (p. 13). But in their study, they had actually relied on social-cognitive models of PTSD (see, e.g., Belsher et al., 2012).

In sum, three points can be made. The first is that FRs are prone to several negative psychological consequences as a result of repeated exposure to harsh circumstances. The second is that there is awareness of these outcomes, and therefore, several treatment approaches have been developed to aid FRs who suffer psychopathological symptoms. The third is that there has been little effort to develop comprehensive health promotion programs for FRs, which could boost their strengths and provide them with generalized and specific resistance resources to help them cope in the face of the mental challenges they confront. Therefore, there seems to be a need for a model of enhancing mental fitness in a salutogenic orientation. Such a model will be offered in the following section.

\section{A Mental Efforts Scale as an Aid in Preparing FRs for Coping with Mental Challenges: Proposing a Salutogenic Mental Fitness Model}

Professional FRs are a subgroup of "exposed populations" groups of people who are repeatedly exposed to aversive details of traumatic events (see PTSD criterion A4) but are not necessarily responders. These include, for example, clerks in criminal courts and administrative workers in emergency rooms or police stations. Whether on the site of an emergency or not, these various populations each have a specific list of tasks they fulfill on their job on a routine basis. Let us take firefighters as an example. When we think of a firefighter, the first thing that probably comes to mind is an image of a person in a protective outfit and a helmet, holding a hose and spraying water on flames. But when we examine carefully what firefighters' work consists of, we come up with a long list of tasks, activities, and situations. Here is a detailed, though not exhaustive, list of examples:

1. Leaving home, wife, and kids early in the morning for a day shift

2. Filling out a written report following a fire call 
3. Having to sleep at the fire station while sharing the room with other people

4. Carrying the weight of the protective suit, helmet, and tools

5. Having to suddenly switch between lazing out at the station and an emergency call

6. Operating close to the heat of a fire

7. Carrying a wounded person out of a burning building

8. Finding the dead body of a person in a fire

9. Finding body remains in a fire

10. Having a buddy killed in a fire

11. Having to face the family of someone who died in a fire

12. Operating while fearing the possibility of getting hurt or dying in a fire

13. Fearing making a fatal operational mistake

14. Taking daily care of firefighting equipment (e.g., extinguishers)

15. Having to pass the minimum score during training and drills

16. Supervising new recruits

17. Operation while having fear of heights

18. Not being able to breath normally during a rescue mission

19. Passing by a house where a fatal fire has once occurred

20. Searching the bedroom of a missing child during a fire

Which of the above is the most stressful task, activity, or situation? It is hard to say. We might intuitively think that tasks involving presence at a fire scene would be much more stressful (e.g., carrying a wounded person out of a burning building) than tasks done at the fire station (e.g., having to share a room with buddies). But we may be wrong. I have learned that sometimes having to sprint "from zero to one hundred" (leaving your dish full of food which had just been prepared at the station, putting on your protective gear, and jumping into the fire engine) is more difficult, mentally, than fighting the fire itself. How can this counterintuitive rating of stress be explained? One possible explanation is that firefighters are trained continuously for fighting fires and rescuing people; firefighters are not usually trained for standing in a safe place away from the fire and talking to a victim's family members, or for maintaining good social relationships with the buddies in their shift. If firefighters are stressed by tasks and situations which are not life-threatening (especially involving developing trust in their chief or buddies), this may have a negative impact on their operational efficiency when they run into a burning house.

The kind of list above can be prepared for other groups of FRs (police investigators, paramedics, etc.). To further elaborate, I will now describe a working model which was developed in the mental fitness branch (established in 2017) of the Department of Health and Well-being of the IDF. The model is still under examination, but so far seems to have promising outcomes. Perhaps, the most distinctive characteristic of the model, in practical terms, is its "tailor-made" nature. While based on the same general salutogenic principles, it is suited differently for every specific kind of exposed population (FRs and others). As an example, I will take the crime scene investigators (CSI) unit in the military police.

Stage 1: Create a list of tasks, activities, and situations

Contact is made with the unit, and we have an informal meeting or a focus group with a small number of soldiers and ask them to describe, in as much detail as possible, all the duties and events they have or may encounter, on their job. We usually end up with a list of 30-60 items. Examples are a search for drugs at someone's home, interrogating suspects of sexual assault, answering a defense attorney's questions in court during cross-examination, lack of sleep, arriving at the scene of an automobile accident, direct exposure to body parts, exposure to difficult life stories, and time management.

Stage 2: Develop a "situation perception" questionnaire

At this stage, we construct a questionnaire to measure soldiers' perceptions of their duties and events in terms of the amount of stress each task or situation elicits, and the amount of confidence they have in performing their duties or coping with the events. Conceptually, it resembles (to a limited extent) the idea of using the critical incident technique (Flanagan, 1954) and qualitative content analysis to develop a job-related self-efficacy scale (see Judge et al., 2007; Lorente et al., 2011). The situation perception questionnaire (SPQ) looks like the example in Table 50.1.

For the CSI unit, we have 36 items. They are ordered randomly to prevent order bias or a halo effect. For each respondent, we calculate the median value for each of the two measures and mark very high-stress scores and very low confidence (or job self-efficacy) scores. We do the same for the unit as a whole. Questionnaires are not anonymous, so we can identify those who have difficulty in specific duties more than in others. We explain the importance of identifying difficulties in order for them to be addressed and treated, and we ensure that the soldiers' commanders do not see the results.

Test-retest correlations we have measured so far are high and are evident of the SPQ's reliability (as stability). There is no point in measuring internal consistency because the questionnaire items are not assumed to measure a single construct. We also measured convergent validity using correlational analyses: we examined the relationship between the perceived stress and the confidence ratings, on the one hand, and other variables such as degree of experience as investigators and SOC, on the other hand. The findings in several units we have worked with so far are consistent: first, there is a very high negative correlation between perceived stress and confidence (or job self-efficacy). Second, the more experienced one is on the job, the less stressed and the more 
Table 50.1 Example of three items in the situation perception questionnaire (SPQ)

\begin{tabular}{|c|c|c|c|c|c|c|c|c|c|c|c|c|c|}
\hline \multirow{3}{*}{1} & \multirow{3}{*}{$\begin{array}{l}\text { Task } \\
\text { Lack of sleep }\end{array}$} & \multicolumn{8}{|c|}{$\begin{array}{l}\text { How much stress do you feel doing the task or } \\
\text { in the situation? }\end{array}$} & \multicolumn{4}{|c|}{$\begin{array}{l}\text { How confident are you in being able to perform the } \\
\text { task or in dealing with the situation? }\end{array}$} \\
\hline & & \multicolumn{4}{|c|}{ No stress } & \multicolumn{4}{|c|}{ Extreme stress } & \multicolumn{2}{|c|}{ Very little } & \multicolumn{2}{|c|}{ Very much } \\
\hline & & 1 & 2 & 3 & 4 & 5 & 6 & 7 & 8 & 1 & 2 & 3 & 4 \\
\hline \multirow[t]{2}{*}{2} & Direct exposure to body parts & \multicolumn{4}{|c|}{ No stress } & \multicolumn{4}{|c|}{ Extreme stress } & \multicolumn{2}{|c|}{ Very little } & \multicolumn{2}{|c|}{ Very much } \\
\hline & & 1 & 2 & 3 & 4 & 5 & 6 & 7 & 8 & 1 & 2 & 3 & 4 \\
\hline \multirow[t]{2}{*}{3} & A search for drugs at someone's & \multicolumn{4}{|c|}{ No stress } & \multicolumn{4}{|c|}{ Extreme stress } & \multicolumn{2}{|c|}{ Very little } & \multicolumn{2}{|c|}{ Very much } \\
\hline & home & 1 & 2 & 3 & 4 & 5 & 6 & 7 & 8 & 1 & 2 & 3 & 4 \\
\hline
\end{tabular}

confident he or she feels (however, this relationship is weak to moderate). Third, SOC is a good predictor of both measures (medium-high negative correlation with perceived stress and medium-high positive correlation with confidence). These repeated results allow us to infer the validity of the SPQ.

\section{Stage 3: Construct and implement a mental efforts scale}

With the results of the questionnaire, we can now order the items according to the degree of perceived stress. We usually do it only at the unit level (i.e., sorted by the median unit score), but when requested by the unit's mental health officer (MHO), we can do it for individual soldiers. Mostly, this is done for soldiers who have a history of meetings with the MHO.

After having a picture of what kinds of tasks, activities, and situations are perceived as less or more stressful, we meet with the unit commanders and offer ways to lessen the pressure when needed. For example, in the case of the CSI unit, they were surprised to find out that being crossexamined by a defense attorney (when investigators were called to testify for the military prosecution) was about as stressful as arriving at a suicide scene. Our recommendation was to train investigators for cross-examination using simulations. Another finding (common in most units) was that tasks involving a great deal of uncertainty (including administrative chores) were experienced as stressful, as well as tasks for which soldiers felt they do not have enough tools or resources. In salutogenic terms, they experienced a lack of comprehensibility and manageability. We recommended that whenever possible, give soldiers as much information you can, or build up uncertainty gradually along the training timeline. Also, make sure they know they have the resources they need (e.g., technological devices or commanders' social support).

This does not require a change in the professional aspects of training. We believe commanders have the knowledge and experience needed for training their soldiers well. We do offer "mental fine-tuning" of the training protocols to overcome mental challenges of which commanders may be unaware. This can be a small change in the way various tasks are taught, or the order in which they are learned, or a change in the general climate.

\section{Stage 4: Offer salutogenically oriented psychoeducational workshops and training techniques}

In addition to mental fine-tuning, we have workshops which offer psychoeducation relevant to the unit's activities, especially those who were found as most stressful for that specific unit. Although during the workshops we do give room for answering questions which usually pertain to psychopathology (such as fear from PTSD), we give more room for discussion and techniques that are aimed at enhancing mental fitness and psychological strengths. Many groups of FRs are nonacademic (although some have earned a bachelor's degree), so we do not present the salutogenic model or SOC in a strict academic manner. Instead, we talk about comprehensibility, manageability, and meaningfulness in a way that will make sense to the FRs. First, we ask what kinds of knowledge and experiences they have, or would like to have, for them to understand what they need to do in various job-related situations as well as in life in general, and what will help them predict how their life (on the job and in general) will look like in the future. Second, we discuss the kinds of resources that are available for them (and those they are missing) to cope with challenges (on the job and in life in general). The kinds of resources may differ from one group of FRs to another, but what is important is that resources are relevant to the challenges; social support is almost always mentioned (and usually described as most important). Third, we discuss the importance of the things they do and the challenges they face (on their job and in life in general) in terms of their meaning and try to find specific factors that create motivation and make coping with the challenges worthwhile.

This discussion of comprehensibility, manageability, and meaningfulness relates to the FRs specific job and life in general. In this way, we keep the workshop directly relevant to their daily work (and to the purpose of having the work- 
shop), and also bring to their awareness, and possibly alter, their thought patterns and general orientation to life. It should be reminded that the soldiers we address are usually 18-20 years old, an age at which the sense of coherence is still in its shaping period.

By raising awareness of the three components of SOC, we cover cognitive, behavioral, and emotional aspects of mental fitness (see definition described earlier), as well as conscious and unconscious processes. The social aspect is touched upon when we speak about manageability, but there is also group work as part of the workshop. Also, we have the participants exercise techniques such as Farchi's Six-C protocol for psychological first aid (Farchi et al., 2018). This protocol was adopted by the IDF (called "Magen," which means "shield" in Hebrew; see Svetlitzky et al., 2019) and later by the US Military (called "iCOVER"; see Adler et al., 2019). An 11-minute video (in English) depicting the iCOVER protocol can be found at https://www.youtube.com/ watch?v=t84_QvbnIT0.

Besides the workshops, and in line with the underlying principle of the iCOVER protocol, neurocognitive training techniques help shift arousal and attention from the amygdala (part of the limbic system, in charge of emotional reactions such as fight, flight, or freeze) to the prefrontal cortex (in charge of rational decision-making). Some of these are known as attention bias modification (ABM) techniques (see Wald et al., 2016). There are also mindfulness workshops and similar sessions to teach relaxation techniques that we employ. All these together are relevant to the physiological aspect of mental fitness.

In addition, we present the salutogenic model and SOC to senior commanders, both as a description of our approach toward mental health promotion and as a new thought paradigm for them to consider. Most of the senior commanders we have met with expressed a positive attitude toward the salutogenic orientation. They have shown interest in mental health promotion activities and the training techniques we employ.

\section{Stage 5: Accompany the intervention with research}

When offering mental fine-tuning in a unit for the first time, we test its efficiency by implementing it in only part of the platoons, while having the others carry out the training as they have done before. We administer the SPQ and the SOC-13 questionnaire at the beginning of training, at its end, and at a follow-up period (usually about 3 months) and compare the change in platoons that received the refined training to the change in those which have not. Unpublished data that have accumulated so far point to the efficacy and usefulness of our model.

\section{Summary and Recommendations}

Research has found FRs to be prone to psychological distress and psychopathology resulting from their repeated exposure to potentially traumatic events. Most of the literature is focused on postevent treatment or on pathologyoriented (preventive) preparation. The mental fitness model proposed earlier, which includes an application of a mental efforts scale accompanied by salutogenically oriented psychoeducation and other activities, is intended to enhance mental fitness among FRs and build their psychological strengths as they face adversities on their job. I believe that having a stronger sense of comprehensibility, manageability, and meaningfulness will enable FRs to cope with such adversities in a way that will reduce the chances of suffering mental injuries, thus preventing the need for postevent treatment. One can say that this does not differ from pathogenically oriented preventive medicine. But this can be said of salutogenesis in general: anything that promotes health thereby prevents disease. However, pathogenesis and salutogenesis are not two sides of the same coin. Disease prevention is indeed a favorable consequence of salutogenic health promotion, but while prevention tends to be diseasespecific (we take a pill to lower blood pressure; we reduce the amount of fat we eat to protect ourselves from heart problems), salutogenic health promotion is holistic in its nature and is targeted for whole populations and not only for individuals at risk.

Therefore, it is recommended that besides psychopathology-oriented programs intended for providing mental first aid to FRs and to the communities who experience potentially traumatic events, there should be more focus on salutogenically based mental preparation programs. These should emphasize the strengths and resources that could help FRs arrive at scenes of disaster equipped with salutogenic resources, at the strategic as well as tactical levels. This way, not only the FR's coping mechanisms will benefit, but they will also better succeed in helping, and even saving, those who need aid and rescue.

Finally, I should add that the idea of salutogenically helping the helpers is not new. After I had finished writing this chapter, I was looking through a box of old papers and found the first page of a lecture given in 1989 in Sweden by Aaron Antonovsky. The lecture was entitled "Caring for the carers," and this was the opening paragraph:

In this lecture, I will attempt to apply what I call the salutogenic model to a crucial problem that is often ignored: the conditions which facilitate the adaptive survival and fulfillment of responsibilities of those who work in the caring professions.

Therefore, I rest my case. 


\section{References}

Adams, J. G., \& Walls, R. M. (2020). Supporting the healthcare workforce during the COVID-19 global epidemic. Journal of the American Medical Association. https://doi.org/10.1001/jama.2020.3972

Adler, A., Start, A. R., Milham, L., Allard, Y. S., Riddle, D., Townsend, L., \& Svetlitzky, V. (2019). Rapid response to acute stress reaction: Pilot test of iCOVER training for military units. Psychological Trauma: Theory, Research, Practice, and Policy. https://doi. org/10.1037/tra0000487

American Psychiatric Association. (2013). Diagnostic and statistical manual of mental disorders (5th ed.). Arlington, VA: Author.

Antonovsky, A. [Aaron]. (1979). Health, stress, and coping. San Francisco: Jossey-Bass.

Antonovsky, A. [Aaron]. (1987). Unraveling the mystery of health: How people manage stress and stay well. San Francisco: Jossey-Bass.

Antonovsky, A. [Avishai], Danon, R., Schiff, M., \& Shelef, L. (2021). Predicting mental burnout among Israeli Home Front Command soldiers during the COVID-19 pandemic. Health Promotion International, 1-13. doi.org/10.1093/heapro/daab036

Antonovsky, A. [Avishai], Svetlitzky, V., Gover, Y., \& Gaffen, M (2017). Definition of mental fitness. Mental Health Department, IDF.

Bandura, A. (1997). Self-efficacy: The exercise of control. New York: Freeman.

Belsher, B. E., Ruzek, J. I., Bongar, B., \& Cordova, M. J. (2012). Social constraints, posttraumatic cognitions, and posttraumatic stress disorder in treatment-seeking trauma survivors: Evidence for a social cognitive processing model. Psychological Trauma: Theory, Research, Practice, and Policy, 4, 386-391.

Bercier, M. L. (2013). Interventions that help the helpers: A systematic review and meta-analysis of interventions targeting compassion fatigue, secondary traumatic stress and vicarious traumatization in mental health workers. Doctoral dissertation, Department of Social Work, Loyola University, Chicago.

Berger, W., Coutinho, E. S. F., Figueira, I., Marques-Portella, C., Luz, M. P., Neylan, T. C., Marmar, C. R., \& Mendlowicz, M. V. (2012). Rescuers at risk: A systematic review and meta-regression analysis of the worldwide current prevalence and correlates of PTSD in rescue workers. Social Psychiatry and Psychiatric Epidemiology, 47, 1001-1011.

Bezabh, Y. H., Abebe, S. M., Fanta, T., Tadese, A., \& Tulu, M. (2017). Prevalence and associated factors of posttraumatic stress disorder among emergency responders of Addis Ababa Fire and Emergency Control and Prevention Service Authority, Ethiopia: Institutionbased, cross sectional study. British Medical Journal Open, e020705. https://doi.org/10.1136/bmjopen-2017-020705

Bodman, G. (2017, November). Nature as health promotion, "The Rug of Life" as a method. Paper presented at the International and Interdisciplinary Conference IMMAGINI? Image and Imagination between Representation, Communication, Education and Psychology, Brixen, Italy.

Bolier, L., Haverman, M., Kramer, J., Westerhof, G. J., Riper, H., Walburg, J. A., Boon, B., \& Bohlmeijer, E. (2013). An internetbased intervention to promote mental fitness for mildly depressed adults: Randomized controlled trial. Journal of Medical Internet Research, 15, 1-18.

Brondolo, E., Kaur, A., Brondolo, T. J., Schwartz, J. E., \& Delahanty, D. L. (2017). Development of a web-based scalable intervention to reduce mental health risks in medical examiner personnel. Testing, Psychometrics, Methodology in Applied Psychology, 24, 1-13.

Brondolo, E., Wellington, R., Brady, N., Libby, D., \& Brondolo, T. J. (2008). Mechanism and strategies for preventing posttraumatic stress disorder in forensic workers responding to mass fatality incidents. Journal of Forensic and Legal Medicine, 15, 78-88.
Brondolo, E., Wellington, R., Brondolo, E., Brondolo, T. J., \& Delahanty, D. (2012). Work-related predictors of psychological distress among medical examiner and coroner personnel. Academic Forensic Pathology, 2, 80-91.

Carleton, R. N., Korol, S., Mason, J. E., Hozempa, K., Anderson, G. S., Jones, N. A., Dobson, K. S., Szeto, A., \& Bailey, S. (2018). A longitudinal assessment of the road to mental readiness training among municipal police. Cognitive Behaviour Therapy, 47, 508-528. https://doi.org/10.1080/16506073.2018.1475504

Chen, Q., Liang, M., Li, Y., Guo, J., Fei, D., Wang, L., He, L., Sheng, C., Cai, Y., Li, X., Wang, J., \& Zhang, Z. (2020). Mental healthcare for medical staff in China during the COVID-19 outbreak. The Lancet Psychiatry, 7(4), e15-e16. https://doi.org/10.1016/ S2215-0366(20)30078-X

Cochran, K., \& Bardi, C. A. (2010). Mental health intervention and prevention strategies for emergency service personnel facing traumatic stress symptoms. Modern Psychological Studies, 16(1), Article 7.

Danon, R., Antonovsky, A. [Avishai], Schiff, M., \& Shelef, L. (2020). Reinforcing the Israeli National Emergency Medical Service by the Home Front Command during the COVID-19 pandemic: The effect of mental health preparation. Military Medicine, 17, 3-13. (Hebrew).

Donnelly, E. (2012). Work-related stress and posttraumatic stress in emergency medical services. Prehospital Emergency Care, 16, 76-85.

Eshel, Y., Kimhi, S., Lahad, M., \& Leykin, D. (2017). Individual attributes as predictors of protective and risk components of resilience under continuing terror attacks: A longitudinal study. Personality and Individual Differences, 114, 160-166.

Farchi, M., Ben Hirsch-Gorneman, M., Bergman Levi, T., Whiteson, A., Ben Gershon, B., \& Gidron, Y. (2018). The SIX Cs model for immediate cognitive psychological first aid: From helplessness to active efficient coping. International Journal of Emergency Mental Health and Human Resilience, 20, 1-12.

Flanagan, J. C. (1954). The critical incident technique. Psychological Bulletin, 51, 327-358.

Hantman, S., \& Farchi, M. (2015). From helplessness to active coping in Israel: Psychological first aid. In E. M. Schott \& E. L. Weiss (Eds.), Transformative social work practice (pp. 467-484). Thousand Oaks, CA: Sage.

Haugen, P. T., McCrillis, A. M., Smid, G. E., \& Nijdam, M. J. (2017). Mental health stigma and barriers to mental health care for first responders: A systematic review and meta-analysis. Journal of Psychiatric Research, 94, 218-229.

Heyman, M., Dill, J., \& Douglas, R. (2018). The Ruderman white paper on mental health and suicide of first responders. Boston, MA: Ruderman Family Foundation.

Judge, T. A., Jackson, C. L., Shaw, J. C., Scott, B. A., \& Rich, B. L. (2007). Self-efficacy and work-related performance: The integral role of individual differences. Journal of Applied Psychology, 92, 107-127.

Kobasa, S. C. (1979). Stressful life events, personality, and health: An inquiry into hardiness. Journal of Personality and Social Psychology, 37, 1-11.

Lahad, M. (2000). Darkness over the abyss: Supervising crisis intervention teams following disaster. Traumatology, 6, 273-293.

Lahad, M. (2017). From victim to victor: The development of the BASIC Ph model of coping and resiliency. Traumatology, 23, 27-34.

Lai, J., Ma, S., Wang, Y., Cai, Z., Hu, J., Wei, N., Wu, J., Du, H., Chen, T., Li, R., Tan, H., Kang, L., Yao, L., Huang, M., Wang, H., Wang, G., Liu, Z., \& Hu, S. (2020). Factors associated with mental health outcomes among healthcare workers exposed to coronavirus disease 2019. Journal of the American Medical Association Network Open, 3(3), e203976-e203976. https://doi.org/10.1001/ jamanetworkopen.2020.3976 
Lorente, L., Salanova, M., \& Martinez, I. (2011). Developing a jobrelated self-efficacy scale among construction workers. Revista Interamericana de Psicología Ocupacional, 30, 149-160.

Ramey, S. L., Perkhounkova, Y., Hein, M., Chung, S., Franke, W. D., \& Anderson, A. A. (2016). Building resilience in an urban police department. Journal of Occupational and Environmental Medicine, $58,796-804$.

Ritchie, H., Hasell, J., Appel, C., \& Roser, M. (2019). Terrorism. Published online at OurWorldInData.org. Retrieved July 23, 2020 from: https://ourworldindata.org/terrorism

Ritchie, H., \& Roser, M. (2019a). Causes of death. Published online at OurWorldInData.org. Retrieved July 23, 2020 from: https://ourworldindata.org/causes-of-death

Ritchie, H., \& Roser, M. (2019b). Natural disasters. Published online at OurWorldInData.org. Retrieved July 23, 2020 from: https://ourworldindata.org/natural-disasters

Robinson, P. L. (2014). Conceptualizing and measuring mental fitness. Doctor of Philosophy thesis, Department of Psychology, University of Wollongong, Australia.

Smith, L. J., Gallagher, M. W., Tran, J. K., \& Vujanovic, A. A. (2018). Posttraumatic stress, alcohol use, and alcohol use reasons in firefighters: The role of sleep disturbance. Comprehensive Psychiatry, 87, 64-71.

Svetlitzky, V., Farchi, M., Ben Yehuda, A., Start, A. R., Levi, O., \& Adler, A. B. (2019). YaHaLOM training in the military: Assessing knowledge, confidence, and stigma. Psychological Services. Advance online publication. https://doi.org/10.1037/ser0000360

Szeto, A., Dobson, K. S., \& Knaak, S. (2019). The road to mental readiness for first responders: A meta-analysis of program outcomes. The Canadian Journal of Psychiatry, 64, 18S-29S.

Tan, B. Y., Chew, N. W., Lee, G. K., Jing, M., Goh, Y., Yeo, L. L., Zhang, K., Chin, H., Ahmad, A., Khan, F. A., Shanmugam, G. N.,
Chan, B. P. L., Sunny, S., Chandra, B., Ong, J. J. Y., Paliwal, P. R., Wong, L. Y. H., Sagayanathan, R., Chen, J. T., ... Sharma, V. K. (2020). Psychological impact of the COVID-19 pandemic on health care workers in Singapore. Annals of Internal Medicine. https://doi. org/10.7326/M20-1083

U.S. First Responders Association. (2019). Who is a first responder? Panama City, FL: Author.

Wald, I., Fruchter, E., Ginat, K., Stolen, E., Dagan, D., Bliese, P. D., Quartana, P. J., Sipos, M. L., Pine, D. S., \& Bar-Haim, Y. (2016). Selective prevention of combat-related posttraumatic stress disorder using attention bias modification training: A randomized controlled trial. Psychological Medicine, 46, 2627-2636.

Weiss, D. S., Brunet, A., Best, S. R., Metzler, T. J., Liberman, A., Pole, M., Fagan, J. A., \& Marmar, C. R. (2010). Frequency and severity approaches to indexing exposure to trauma: The critical incident history questionnaire for police officers. Journal of Traumatic Stress, 23, 734-743. https://doi.org/10.1002/jts.20576

Windle, G. (2011). What is resilience? A review and concept analysis. Reviews in Clinical Gerontology, 21, 12-169.

World Health Organization. (2018). Global status report on road safety 2018. Geneva: Author.

Zamorski, M. (2008). Defining resilience: An international perspective [PowerPoint slides]. Retrieved July 23, 2020 from http://www.imta. info/PastConferences/Presentations.aspx?Show $=2008$

Zerach, G., \& Levin, Y. (2015). Posttraumatic stress symptoms, burn-out, and compassion satisfaction among body handlers: The mediating role of sense of coherence and spirituality at workplace. Journal of Interpersonal Violence, 1-27. https://doi. org/10.1177/0886260515621065

Open Access This chapter is licensed under the terms of the Creative Commons Attribution 4.0 International License (http://creativecommons. org/licenses/by/4.0/), which permits use, sharing, adaptation, distribution and reproduction in any medium or format, as long as you give appropriate credit to the original author(s) and the source, provide a link to the Creative Commons license and indicate if changes were made.

The images or other third party material in this chapter are included in the chapter's Creative Commons license, unless indicated otherwise in a credit line to the material. If material is not included in the chapter's Creative Commons license and your intended use is not permitted by statutory regulation or exceeds the permitted use, you will need to obtain permission directly from the copyright holder. 